\title{
S194-P-FADD as a marker of aggressiveness and poor prognosis in human T-cell lymphoblastic lymphoma.
}

SHORT TITLE: FADD phosphorylation in human T-LBL.

José L Marín-Rubio ${ }^{1,2,3}$, Eduardo Pérez-Gómez ${ }^{4,5}$, José Fernández-Piqueras ${ }^{1,2,3,6,{ }^{+}, *}$ and María VillaMorales ${ }^{1,2,3,6,+, *}$.

${ }^{1}$ Departamento de Biología, Universidad Autónoma de Madrid, Madrid, 28049, Spain.

${ }^{2}$ Centro de Biología Molecular Severo Ochoa (CBMSO), Madrid, 28049, Spain.

${ }^{3}$ IIS-Fundación Jiménez Díaz, Madrid, 28040, Spain.

${ }^{4}$ Departamento de Bioquímica y Biología Molecular, Universidad Complutense de Madrid, Madrid, 28040, Spain.

${ }^{5}$ Instituto de Investigación Hospital 12 de Octubre, Madrid, 28041, Spain.

${ }^{6}$ Centro de Investigaciones Biomédicas en Red de Enfermedades Raras (CIBERER), Madrid, 28029 Spain.

* To whom correspondence should be addressed. Tel: +34 911964653; Fax: +34 911964420; Email: maria.villa@uam.es, mvilla@cbm.csic.es.

Correspondence may also be addressed to José Fernández-Piqueras, Tel: +34 911964627; Fax: +34 911964420; Email:jfpiqueras@cbm.csic.es.

${ }^{\dagger}$ These authors contributed equally to this work.

(C) The Author(s) 2019. Published by Oxford University Press. All rights reserved. For Permissions, please email: journals.permissions@oup.com. 


\section{Abstract}

T-cell lymphoblastic lymphoma is a haematological disease with an urgent need for reliable prognostic biomarkers that allow therapeutic stratification and dose adjustment. The scarcity of human samples is responsible for the delayed progress in the study and the clinical management of this disease, especially compared to T-cell acute lymphoblastic leukaemia, its leukemic counterpart. In the present work, we have determined by immunohistochemistry that S194-P-FADD protein is significantly reduced in a cohort of 22 samples from human T-cell lymphoblastic lymphoma. Notably, the extent of such reduction varies significantly among samples and has revealed determinant for the outcome of the tumour. We demonstrate that FADD phosphorylation status impacts on protein stability, sub-cellular localization and non-apoptotic functions, specifically cell proliferation. Phosphorylated FADD would be more stable and preferentially localized to the cell nucleus; there, it would favour cell proliferation. We show that patients with higher levels of S194-P-FADD exhibit more proliferative tumours and that they present worse clinical characteristics and a significant enrichment to an oncogenic signature. This supports that FADD phosphorylation may serve as a predictor for T-cell lymphoblastic lymphoma aggressiveness and clinical status. In summary, we propose FADD phosphorylation as a new biomarker with prognostic value in Tcell lymphoblastic lymphoma. 


\section{Summary}

This study identifies S194-P-FADD as an unfavourable clinical factor in T-cell lymphoblastic lymphoma patients.

Thus, we propose FADD phosphorylation as a new biomarker with prognostic value in T-LBL. 


\section{Introduction}

Precursor T-cell lymphoblastic neoplasms are aggressive haematological malignancies most often manifesting with extensive marrow and blood affectation [acute T-cell lymphoblastic leukaemia, T-ALL], and less commonly as a mass lesion in the thymus/anterior mediastinum or in lymph nodes, with less than $25 \%$ marrow blasts [T-cell lymphoblastic lymphoma, T-LBL] [1]. The molecular basis of these neoplasms has been well established in T-ALL [2-4], but to a lesser extent in T-LBL [5, 6], mainly due to the scarcity of samples.

Current therapies for T-LBL have steadily improved; however, they present two major weaknesses [5]: acute long-term toxicity of the current treatments and survival rates inferior to $10 \%$ at relapse. Both reasons argue for the urgent need to obtain prognostic markers enabling the therapeutic stratification of patients. No reliable prognostic markers are currently available for T-LBL, in contrast to what happens with T-ALL [7]. In T-ALL, the presence of minimal residual disease [MRD] is highly predictive, but to extrapolate such MRD-based stratification to T-LBL patients is technically difficult $[8,9]$. Other risk factors have been postulated [10-12], but more robust prognostic models are required [7].

The oncogenic character of FADD has been extensively described, mainly due to its canonical function as an adaptor for every known death receptor to transmit their apoptotic signals [13]. Cancer cells frequently become resistant to a possible apoptotic insult; such resistance may be acquired by means of different mechanisms, among them FADD downregulation [14, 15]. Besides this canonical function in apoptosis, increasing evidence indicate that FADD is relevant for non-apoptotic functions in T cells [13]; FADD would be essential at early stages of haematopoiesis $[16,17]$ and it has been postulated as critical at the pre-TCR checkpoint of T-cell progenitors, not only signalling apoptosis when they fail but driving proliferation when they success [18]. FADD ability to promote the progression of T cells through the cell cycle would depend on its phosphorylation status $[19,20]$. Moreover, the sub-cellular localization of FADD also seems to be dependent on its phosphorylation; not only are the nuclear localization 
sequence and the nuclear export sequence required for nuclear-cytoplasmic transport of FADD, but also the specific phosphorylation of the protein at serine 194 [20-23].

Nevertheless, the exact mechanisms whereby phosphorylated FADD exerts its non-apoptotic functions and how this might contribute to cancer are not clear. In a recent study in mouse T-LBL [24], we observed that FADD phosphorylation correlated with tumour cell proliferation and tumour aggressiveness, positioning FADD phosphorylation as a putative prognostic marker to study. To determine if FADD phosphorylation is a relevant factor at risk assessment of human T-LBL would help to improve the prognosis of this disease and to propose new targeted therapeutic strategies.

This is the first study, to our knowledge, that identifies S194-P-FADD as an unfavourable clinical factor in T-LBL patients; moreover, we unravel that increased tumour cell proliferation is the molecular basis underlying this clinical effect. Altogether, the results presented in this study support to consider S194-P-FADD as a new biomarker with prognostic value, which may allow for therapeutic stratification of T-LBL patients.

\section{Materials and methods}

Human samples

Twenty-two human T-LBL samples and twenty-four human healthy thymus samples were obtained through the Spanish Tumour Bank Network and Hospital de La Paz, respectively. The source of origin of T-LBL samples were either mass lesions in mediastinum or lymph nodes, independently of age. Regarding the control healthy thymuses, they were all paediatric. Institutional review board approval was obtained for these studies (CEI-70-1260) and the participants provided written informed consent in accordance with the Declaration of Helsinki.

Cell culture 
JURKAT clone A3 (ATCC Cat\# CRL-2570, RRID:CVCL_1061) and FADD-deficient JURKAT clone I2.1 (ATCC Cat\# CRL-2572, RRID:CVCL_3724) were purchased in 2015 and HEK-293T (ATCC Cat\# CRL11268, RRID:CVCL_1926) was purchased in 2013, all from ATCC (Manassas, VA, USA). JURKAT cell lines were cultured in RPMI 1640 and HEK-293T cells were cultured in DMEM, both from Gibco (Life Technologies, Carlsbad, CA, USA); media were supplemented with $15 \%$ and $10 \%$ FBS, respectively (GE Healthcare Life Sciences, Velizy-Villacoublay, France), 2 mM L-Glutamine (Merck Millipore, Billerica, MA, USA) and $1 \mathrm{mM}$ sodium pyruvate (Merck Millipore). Cultures were maintained at $37^{\circ} \mathrm{C}$ in $5 \% \mathrm{CO} 2$ humidified atmosphere. ATCC routinely performs cell lines authentication, using STR profiling as a procedure. Cell experimentation was always performed within a period not exceeding 6 months after resuscitation and in mycoplasma-free culture conditions.

Generation of cell lines stably expressing FADD

JURKAT I2.1 cell line was transduced with lentiviral particles carrying EX-V0108-Lv-225 (FADD cDNA), its empty control EX-NEG-Lv225 (GeneCopoeia, Rockville, MD, USA) or S194A-FADD- and S194DFADD-derived vectors, obtained after HEK-293T- mediated packaging using pMD2.G and psPAX2 (Addgene, Cambridge, MA, USA). Puromycin was added at $2 \mu \mathrm{g} / \mathrm{ml}$ for selection and at $3 \mu \mathrm{g} / \mathrm{ml}$ for maintenance. Genetic modification of JURKAT I2.1 cell line by lentiviral transduction was subjected to the regulated and approval by the Spanish Ministry of Agriculture, Food, and Environment, managed by CBMSO's Biological Safety Department. We performed site-directed mutagenesis to obtain S194AFADD- and S194D-FADD-derived vectors, using the QuikChange Lightning Site-Directed Mutagenesis Kit (Agilent Technologies, Santa Clara, CA, USA) and following the manufacturer instructions. The oligonucleotide primers used for site-directed mutagenesis are (the mutated sequences are underlined): Ser194Ala [S194A]: forward: 5'catgacatcggggccatggccccactc 3', reverse: 5'gagtggggccatggccccgatgtcatg 3'; Ser194Asp [S194D]: forward: 5'gttccatgacatcgggtccatggccccactcctg 3', reverse: 5'caggagtggggccatggacccgatgtcatggaac 3'. 
Antibodies and reagents

Antibodies used for immunodetection are summarized in Supplementary Table 1. Cycloheximide [CHX], anisomycin and nocodazole were purchased from Sigma-Aldrich (Sigma-Aldrich, St. Louis, MO, USA) and MG-132 from PeptaNova (PeptaNova, Sandhausen, Germany). 
Immunohistochemistry [IHC] / Immunocytochemistry [ICC]

Paraffin-embedded tissues were deparaffinised and rehydrated using standard protocols and subjected to heat-induced antigen retrieval in Tris-EDTA buffer $(\mathrm{pH}=9.0)$ or sodium citrate buffer with $0.05 \%$ Tween 20 $(\mathrm{pH}=9.0)$. Frozen tissues in Tissue-Tek® O.C.T ${ }^{\mathrm{TM}}$ Compound (Sakura Finetek Europe B.V., Alphen aan den Rijn, The Netherlands) were fixed with $10 \%$ formalin for 10 min. For ICC, the cells were washed twice with TBS $1 \mathrm{X}$ before being frozen in Tissue-Tek® O.C. T $^{\mathrm{TM}}$ Compound. Dako REAL ${ }^{\mathrm{TM}}$ antibody diluent (Dako, Glostrup, Denmark) was the blocking buffer. We used liquid DAB+ substrate chromogen system (Dako) for visualization. Olympus BX61 microscope (Olympus Corporation of the Americas, Center Valley, PA, USA) at 40x or 100x magnification was used for image analysis. Irrespective of intensity, the percentages of positive cells versus total cell number were calculated using at least five random representative fields per section. Fiji-Image $\mathrm{J}$ free software (ImageJ, RRID:SCR_003070) (National Institutes of Health, Bethesda, MD, USA) was used for analysis, applying the Image/Colour deconvolution, Image/Adjust/Threshold and Analyse/Analyse Particles tools as published previously [25].

Western blot [WB]

Total proteins were obtained using RIPA cell lysis buffer. Nuclear and cytoplasmic fractionated protein extracts were obtained using cytoplasmic extract buffer (10 mM HEPES, $60 \mathrm{mM} \mathrm{KCl,} 1 \mathrm{mM}$ EDTA with $0.075 \% \mathrm{NP}-40$ ) and RIPA. Protein extracts were supplemented with $2 \mathrm{mM} \mathrm{PMSF}, 2.5 \mu \mathrm{l} / \mathrm{ml}$ Protease Inhibitor Cocktail and $10 \mu \mathrm{l} / \mathrm{ml}$ Phosphatase Inhibitor Cocktail 2 (Roche Diagnostics GmbH, Mannheim, Germany). Ten micrograms-aliquots were electrophoresed in Mini-PROTEAN-TGX ${ }^{\mathrm{TM}}$ Precast Gels (BioRad Laboratories, Hercules, CA, USA) and then electrotransferred to mini-sized PVDF membranes by Transfer Blot® Turbo $^{\mathrm{TM}}$ Transfer System (Bio-Rad Laboratories). The peroxidase activity was developed using WesternBright ECL Detection System (Advansta, Menlo Park, CA, USA). ImageQuant LAS 4000 digital imaging system (GE Healthcare Europe GmbH, Freiburg, Germany) was used for acquisition of 
images, and Quantity One® v4.6.3 (Quantity One 1-D Analysis Software, RRID:SCR_014280) (Bio-Rad Laboratories) for band densitometry. 
Determination of protein stability

For protein stability analysis, cells were treated with $\mathrm{CHX}$, anisomycin and/or MG-132, as indicated. Protein levels were determined at $0,2,4$, and $6 \mathrm{~h}$ after treatment by WB.

Gene set enrichment analysis [GSEA]

GSEA was performed using the GSEA-R, a Bioconductor implementation of GSEA (Gene Set Enrichment Analysis, RRID:SCR_003199) from Broad Institute [26]. Analysis was run with 1000 permutations and a classic statistic. Normalized enrichment score and nominal P value were measured.

In-vitro functional assays

Cell viability was assessed during 5 days using the Cell Proliferation Kit II (XTT) (Sigma-Aldrich), following manufacturer's instructions.

Mitotic cells number was determined by flow cytometry $[\mathrm{FC}]$ as the percentage of cells positive for phosphorylated histone H3 staining, using Alexa Fluor®488-conjugated phospho-Histone H3 (Ser10) Antibody (Cell Signaling Technology Cat\# 9708, RRID:AB_331567) (Danvers, MA, USA) and a FACS Canto II (Becton-Dickinson, Franklin Lakes, NJ, USA).

Time for completion of mitosis was estimated by FC as the ratio $2 n / 4 n$ DNA cell content at specific time points after G2/M-synchronization by nocodazole treatment and release, using PI/RNase Staining Buffer (BD Biosciences, San Jose, CA, USA). Time points were obtained by harvesting cells every 20 min after release.

Apoptosis was monitored both by WB-immunodetection of caspases activation and by FC using the PE Annexin-V Apoptosis Detection Kit I (BD Biosciences) after 24 h-treatment of $10^{6} \mathrm{cells} / \mathrm{ml}$ with agonist anti-FAS mouse antibody clone CH11 (Millipore Cat\# 05-201, RRID:AB_309653) (Merck Millipore) or with 
irrelevant IgM $\lambda$ Mouse IgM Isotype Control (11E10), eBioscience ${ }^{\mathrm{TM}}$ (Thermo Fisher Scientific Cat\# 144752-81, RRID:AB_470122) (Invitrogen, Carlsbad, CA, USA) at a final concentration of $100 \mathrm{ng} / \mathrm{ml}$.

Results from FC were analysed using FlowJo v10 (FlowJo, RRID:SCR_008520) (Ashland, OR, USA). 
Statistical analyses

The Kolmogorov-Smirnov test was used to check expression data sets for normality and the Levene test was used for homogeneity of variances, using SPSS (SPSS, RRID:SCR_002865) (IBM Corporation, Somers, NY, USA). Significances between two or more groups were determined by Mann-Whitney $U$ or Kruskal-Wallis test, respectively. Kaplan-Meier survival curves were performed by the Mantel-Cox method, using the GraphPad Prism 6 (GraphPad Prism, RRID:SCR_002798) (GraphPad Software, La Jolla, CA, USA). Kernel density plot was performed with R v3.2.3 (R Project for Statistical Computing, RRID:SCR_001905).

\section{Results}

Human samples of T-LBL can be stratified depending on FADD levels

RNA-sequencing analysis had been carried out in a discovery cohort consisting of two control foetal thymuses and eight T-LBL samples (GSE109234); our results indicated that $37,5 \%$ of the samples (3/8) exhibited FADD downregulation, corroborating our previous observation that $35 \%$ of human T-LBL cases exhibited reduced FADD levels [27].

To validate these results at the protein level, we performed FADD IHC in an extended cohort of 24 control samples from paediatric thymuses and 22 T-LBL samples. Our results evidenced a significant reduction of FADD in the T-LBL group in comparison to control healthy thymuses (Figure 1A, left); however, a considerable inter-tumour heterogeneity was observed among the T-LBL samples. A Gaussian Kernel density plot showed a skewed distribution of the samples in two clusters with different levels of FADD positivity by IHC (Figure 1A, right). On the basis of these clusters, two T-LBL sub-groups were defined, namely High and Low (Figure 1B). FADD reduction was not due to the presence of mutations, as it was corroborated by FADD gene sequencing (data not shown). 
S194-P-FADD was also immunostained in the same cohort (Figure 1C), which also revealed a significant reduction of S194-P-FADD positivity in T-LBL compared to the control group; most interestingly, the comparison of S194-P-FADD levels between the High and Low T-LBL sub-groups evidenced a significant distinction that further supported this clustering.

\section{S194-P-FADD preferentially localizes to the nuclei of tumour T cells}

We compared the levels of S194-P-FADD in cytoplasms and nuclei of tumour cells from T-LBL samples belonging to High and Low sub-groups (Figure 2A). The distribution of the samples in these two groups was consistent when the subcellular compartments were observed, being the differences between groups statistically significant both for cytoplasmic and nuclear staining. Of note, we observed that, for every group, S194-P-FADD positivity was significantly higher in the nuclei than in the cytoplasms, arguing for a preferential localization of the phosphorylated protein in the nucleus of the tumour cell, where it might exert non-apoptotic functions.

To further evaluate this fact, we developed an in-vitro model of FADD presence and phosphorylation status. Four different JURKAT cell lines were established by lentiviral transduction of the

FADD-deficient clone 12.1; one cell line stably expresses an empty vector, thus being still deficient for FADD [NEG], while the others stably express wild-type FADD [FADD], non-phosphorylatable mutant FADD [S194A] or phosphomimetic mutant FADD [S194D]. Clones selection was made on the basis of similar percentage and intensity of GFP signal and pertinent expression of FADD protein; additionally, we corroborated that FADD expression of stable cell lines was equivalent to FADD endogenous level of the parental clone JURKAT A3 (Supplementary Figure 1). We performed FADD immunodetection in soluble (cytoplasmic) and insoluble (nuclear) protein fractions obtained from these cell lines (Figure 2B). The two bands corresponding to phosphorylated and unphosphorylated wildtype FADD, as well as S194A and S194D FADD proteins, were present in the two compartments. FADD intensity in the nucleus fraction was significantly higher in S194D extract than in the rest, suggesting that nuclear localization of FADD might be favoured by its phosphorylated status. 
We verified this result qualitatively by anti-FADD ICC in the cell lines, which showed more FADDpositive nuclei in S194D cell line than in S194A or FADD cell lines (Supplementary Figure 2). Altogether, these observations make it reasonable to presume that FADD protein can occupy both cell compartments - cytoplasm and nucleus - but the phosphorylated form favours the nuclear localization. 
FADD phosphorylation influences protein stability in tumour T cells

According to previous studies [28], serine-phosphorylation may affect protein stability and, in turn, protein function. To determine if such is the case for FADD, the cell lines FADD, S194A and S194D were treated with the protein synthesis inhibitor $\mathrm{CHX}$, in order to compare FADD protein stability (Figure 3A). Our results revealed that S194D FADD protein is remarkably more stable with time than wild-type FADD or S194A FADD, suggesting that phosphorylation at Ser194 positively affects FADD protein stability. We treated the cells with the proteasome inhibitor MG-132 in combination with $\mathrm{CHX}$, which rescued the reduction of FADD levels, thus indicating that most of protein loss occurred via proteasomal degradation (Figure 3B). These results were corroborated using anisomycin, a different inhibitor of protein synthesis (Supplementary Figure 3 A, B).

FADD phosphorylation favours tumour T cell proliferation

A previous observation in mouse T-LBL indicated that reduced levels of S191-P-FADD in the nucleus of tumour T cells inversely correlated with the proliferation capacity of such cells [24]. Following this idea, we have evaluated Ki67 immunostaining as a marker for proliferation in our cohort of human T-LBL samples. Increased cell proliferation is a common characteristic of tumour cells and such was the case of T-LBL samples in this cohort; notwithstanding, the proliferation rate measured by Ki67-positivity was significantly higher in the High T-LBL sub-group than in the Low T-LBL sub-group (Figure 4A). In addition, GSEA analysis identified a significant enrichment for a signature of T-cell proliferation in the High T-LBL subgroup (Figure 4B). Moreover, a regression analysis showed a significant correlation between Ki67 and nuclear-S194-P-FADD (Figure 4C). Altogether, these results indicate that tumour cell proliferation is favoured by FADD phosphorylation in human T-LBL.

To prove the functional link between FADD phosphorylation and tumour T cell proliferation, in vitro assays were performed. First, the numbers of viable cells were determined in cell lines expressing wildtype FADD or S194D / S194A mutant FADD, in comparison with FADD-deficient cell line, indicating 
that cell proliferation was favoured by FADD presence, particularly in cells expressing the phosphomimetic mutant FADD [S194D] (Figure 5A). S10-P-Histone H3 evaluation as a marker for late G2/M [29] provided the same result, with the S194D cell line exhibiting a significantly higher percentage of cells in mitosis (Figure 5B). On a different approach, G2/M-synchronization by nocodazole treatment and release revealed that the $2 n / 4 n$ ratio increased significantly faster for S194D cell line than for the rest (Figure 5C). This is consistent with a faster completion of mitosis for cells expressing S194D FADD, which may contribute to increased proliferation. Apoptosis assays demonstrated that increased cell viability in S194D was not due to apoptosis impairment (Supplementary Figure 4), further supporting that FADD phosphorylation favours tumour T cell proliferation.

FADD phosphorylation correlates with clinical markers of aggressiveness and unfavourable prognosis

In our cohort of $22 \mathrm{~T}$-LBL samples, we managed to collect the clinical data from 18 patients (Supplementary Table 2). So far, our results suggested that FADD phosphorylation favours nuclear localization, increased stability of the protein and tumour cell proliferation. Thus, we aimed to assess whether FADD phosphorylation level has clinical implications. Unfortunately, and given the scarcity of TLBL samples, the most obvious comparison of disease-free survival and overall survival in High and Low T-LBL sub-groups produced no significant results (data not shown). However, we calculated the mean value of S194-P-FADD positivity of the patients belonging to each clinical category and made the appropriate comparisons (Supplementary Table 2). Very interesting results emerged from this analysis: S194-P-FADD values showed no dependency on sex, age, predominant population in the tumour or immunophenotype. Significant differences existed, however, regarding parameters associated with the aggressiveness and the outcome of the tumour (Supplementary Table 2 and Figure 6A); patients belonging to stage IV exhibited S194-P-FADD values significantly higher than those of stage I. Accordingly, FADD phosphorylation was significantly higher in those patients with involvement of extralymphatic organs. And most interestingly, those patients not overcoming the disease (progressive) or suffering relapse (recurrent) had significantly higher levels of S194-P-FADD, compared to patients without 
relapse. In support of these results, GSEA analysis identified a significant enrichment of an oncogenic signature in the High T-LBL sub-group, compared to the Low (Figure 6B and Supplementary Table 3). Together, these observations indicate that S194-P-FADD level significantly correlates with clinical parameters of tumour aggressiveness and poor prognosis, strongly suggesting that FADD phosphorylation status may serve as a new biomarker with predictive value.

\section{Discussion}

The role of FADD in cancer is controversial. An increase of FADD associated with poor clinical outcome like drug resistance, inferior survival, more recurrence or metastasis has been described in many solid tumours [30-37]. However, a reduction of FADD has been reported as well [38-40]. Such apparent discrepancies between studies could be explained by the fact that FADD exhibits apoptotic and nonapoptotic roles, so the balance between loss and gain of these functions could be dependent on cell type and would determine the final outcome of each tumour [41]. Regarding T-cell lymphoblastic lymphoma, our previous results in mice evidenced a significant reduction of FADD that correlated with impaired apoptosis in tumour cells [24]. In the present study, we observe a significant reduction of FADD protein levels in a cohort of 22 T-LBL human samples, compared to 24 control healthy thymuses. Our T-LBL samples present a virtual absence of active caspase-3 as a marker for apoptosis (IHC-positivity inferior to $2 \%$ in all samples; data not shown). Since the major role of FADD is to be an adaptor for every known death receptor [13], apoptosis impairment appears as a plausible consequence of FADD reduction in cancer, as previously indicated [14].

However, apart from the major role of FADD in apoptosis, evidence compiled in the recent years indicate that FADD protein can also induce survival, cell cycle progression and cell proliferation, depending on its phosphorylation status and subcellular localization [13]. FADD phosphorylation has been analysed in numerous solid tumours, either resulting reduced [35, 42, 43] or increased [31], in all cases associated with poor outcome. Very limited and heterogeneous results are, however, available in haematological human cancers $[44,45]$. In the present study with human T-LBL, we observe a global reduction of S194-P-FADD, but most interestingly, two distinct groups appear within the T-LBL cohort - 
namely High and Low - that exhibit a clear difference of S194-P-FADD levels. Our results suggest that phosphorylated FADD would be more stable, which is supported by previous studies in other cancer types [46]. Besides, a preferential localization of S194-P-FADD is observed in the nucleus of tumour T cells, as it has been previously reported in other tumour types [33]. In the nucleus, it has been hypothesized that FADD would be prevented from participating in death receptors-mediated apoptosis [47] and it would favour tumour cell proliferation [13]. Our previous results in murine T-LBL demonstrated that FADD phosphorylation level correlated with the proliferation capacity of the tumour cells [24]. This has been corroborated in human T-LBL in the present study; not only are the patient samples in the High group significantly more proliferative, but they show a significant association between S194-P-FADD levels and T-cell proliferation, both by Ki67 immunostaining and by GSEA analysis. In vitro assays support that FADD phosphorylation does not prevent apoptosis in the tumour $\mathrm{T}$ cell, thus increasing its survival, but it actively favours tumour T cell proliferation.

Most interestingly, our results evidence that this may have clinical implications; higher levels of S194-P-FADD significantly associate to clinical parameters of tumour aggressiveness and unfavourable prognosis in the patients, as well as an enrichment in an oncogenic signature, strongly suggesting that FADD phosphorylation status may serve as a new biomarker with predictive value.

The main conclusions of this study are summarized in the following model (Figure 6C): the tumour cells of T-LBL patients diagnosed with higher levels of S194-P-FADD will be more proliferative, rendering the tumour more aggressive and impacting on patient prognosis. Thus, FADD phosphorylation status may help the therapeutic stratification of T-LBL patients and shall be considered for biomarker with prognostic value in T-LBL. Further studies on S194-P-FADD levels in larger series of T-LBL samples - and also in other haematological malignancies - are required in order to refine its use as a reliable biomarker.

\section{Supplementary material}

Supplementary Figures 1 to 4 and Supplementary Tables 1 to 3 can be found at http://carcin.oxfordjournals.org/ 


\section{Funding}

This work was supported by Spanish Ministry of Economy and Competitiveness (SAF2015-70561 MINECO/FEDER UE to J.F.-P./M.V.-M., BES-2013-065740 to J.F.-P., PI17/00041 MINECO/FEDER to E.P.-G.); Autonomous Community of Madrid, Spain (B2017/BMD-3778 LINFOMAS-CM to J.F.-P.); Scientific Foundation of Spanish Association Against Cancer (AECC 2018 PROYE18054PIRI to J.F.-P., AECC 2017/0110 to E.P.-G.); and Instituto de Salud Carlos III (ACCI-CIBERER-17 ISCIII to J.F.-P.). J.L.M.-R. is the recipient of a predoctoral fellowship from Spanish Ministry of Education, Culture and Sports (FPU13/00338). Institutional grants from Fundación Ramón Areces and Banco de Santander to CBMSO are also acknowledged.

\section{Acknowledgements}

We thank Ma Ángeles Cobos Fernández, Isabel Sastre and María C. de Arriba for technical help, the Flow Cytometry services at CBMSO and the Histology facility at Centro Nacional de Biotecnología (CSIC). We specially thank the patients who were willing to donate their samples, otherwise this research would not be possible.

Conflict of Interest Statement: None declared.

\section{References}

1. de Leval, L., et al. (2009) Molecular classification of T-cell lymphomas. Crit Rev Oncol Hematol, 72(2): 125-43.

2. Aifantis, I., et al. (2008) Molecular pathogenesis of T-cell leukaemia and lymphoma. Nat Rev Immunol, 8(5): 380-90.

3. Belver, L., et al. (2016) The genetics and mechanisms of T cell acute lymphoblastic leukaemia. Nat Rev Cancer, 16(8): 494-507. 
4. Van Vlierberghe, P., et al. (2012) The molecular basis of T cell acute lymphoblastic leukemia. $J$ Clin Invest, 122(10): 3398-406.

5. Bonn, B.R., et al. (2013) Incidence and prognostic relevance of genetic variations in T-cell lymphoblastic lymphoma in childhood and adolescence. Blood, 121(16): 3153-60.

6. Burkhardt, B. (2010) Paediatric lymphoblastic T-cell leukaemia and lymphoma: one or two diseases? Br J Haematol, 149(5): 653-68.

7. Cortelazzo, S., et al. (2017) Lymphoblastic lymphoma. Crit Rev Oncol Hematol, 113: 304-17.

8. Callens, C., et al. (2012) Clinical impact of NOTCH1 and/or FBXW7 mutations, FLASH deletion, and TCR status in pediatric T-cell lymphoblastic lymphoma. J Clin Oncol, 30(16): 1966-73.

9. Hoelzer, D., et al. (2016) Acute lymphoblastic leukaemia in adult patients: ESMO Clinical Practice Guidelines for diagnosis, treatment and follow-up. Ann Oncol, 27(suppl 5): v69-v82.

10. Balbach, S.T., et al. (2016) Proposal of a genetic classifier for risk group stratification in pediatric T-cell lymphoblastic lymphoma reveals differences from adult T-cell lymphoblastic leukemia. Leukemia, 30(4): 970-3.

11. Beldjord, K., et al. (2014) Oncogenetics and minimal residual disease are independent outcome predictors in adult patients with acute lymphoblastic leukemia. Blood, 123(24): 3739-49.

12. Bonn, B.R., et al. (2015) Whole exome sequencing hints at a unique mutational profile of paediatric T-cell lymphoblastic lymphoma. Br J Haematol, 168(2): 308-13.

13. Tourneur, L., et al. (2010) FADD: a regulator of life and death. Trends Immunol, 31(7): 260-9.

14. Peter, M.E., et al. (2015) The role of CD95 and CD95 ligand in cancer. Cell Death Differ, 22(5): 885-6.

15. Villa-Morales, M., et al. (2012) Targeting the Fas/FasL signaling pathway in cancer therapy. Expert Opin Ther Targets, 16(1): 85-101. 
16. Rosenberg, S., et al. (2011) FADD deficiency impairs early hematopoiesis in the bone marrow. $J$ Immunol, 186(1): 203-13.

17. Zhang, H., et al. (2011) Functional complementation between FADD and RIP1 in embryos and lymphocytes. Nature, 471(7338): 373-6.

18. Newton, K., et al. (2000) FADD/MORT1 regulates the pre-TCR checkpoint and can function as a tumour suppressor. Embo J, 19(5): 931-41.

19. Hua, Z.C., et al. (2003) A function of Fas-associated death domain protein in cell cycle progression localized to a single amino acid at its C-terminal region. Immunity, 18(4): 513-21.

20. Screaton, R.A., et al. (2003) Fas-associated death domain protein interacts with methyl-CpG binding domain protein 4: a potential link between genome surveillance and apoptosis. Proc Natl Acad Sci U S A, 100(9): 5211-6.

21. Gomez-Angelats, M., et al. (2003) Molecular evidence for the nuclear localization of FADD. Cell Death Differ, 10(7): 791-7.

22. Ramos-Miguel, A., et al. (2011) Correlation of rat cortical Fas-associated death domain (FADD) protein phosphorylation with the severity of spontaneous morphine abstinence syndrome: role of alpha(2)-adrenoceptors and extracellular signal-regulated kinases. J Psychopharmacol, 25(12): 1691-702.

23. Zhang, J., et al. (2004) FADD and its phosphorylation. IUBMB Life, 56(7): 395-401.

24. Marin-Rubio, J.L., et al. (2016) Deregulated FADD expression and phosphorylation in T-cell lymphoblastic lymphoma. Oncotarget, 7(38): 61485-99.

25. Ruifrok, A.C., et al. (2001) Quantification of histochemical staining by color deconvolution. Anal Quant Cytol Histol, 23(4): 291-9. 
26. Subramanian, A., et al. (2005) Gene set enrichment analysis: a knowledge-based approach for interpreting genome-wide expression profiles. Proc Natl Acad Sci U S A, 102(43): 15545-50.

27. Villa-Morales, M., et al. (2014) FAS system deregulation in T-cell lymphoblastic lymphoma. Cell Death Dis, 5: e1110.

28. Chen, X., et al. (2012) Serine 123 phosphorylation modulates p21 protein stability and activity by suppressing ubiquitin-independent proteasomal degradation. J Biol Chem, 287(41): 34410-8.

29. Sun, A., et al. (2012) Level of phosphohistone $\mathrm{H} 3$ among various types of human cancers. BMJ Open, 2(5).

30. Callegari, C.C., et al. (2016) Copy number and expression analysis of FOSL1, GSTP1, NTSR1, FADD and CCND1 genes in primary breast tumors with axillary lymph node metastasis. Cancer Genet, 209(7-8): 331-9.

31. Chen, G., et al. (2005) Phosphorylated FADD induces NF-kappaB, perturbs cell cycle, and is associated with poor outcome in lung adenocarcinomas. Proc Natl Acad Sci U S A, 102(35): 12507-12.

32. Chien, H.T., et al. (2016) Clinical Implications of FADD Gene Amplification and Protein Overexpression in Taiwanese Oral Cavity Squamous Cell Carcinomas. PLoS One, 11(10): e0164870.

33. Gibcus, J.H., et al. (2007) Amplicon mapping and expression profiling identify the Fas-associated death domain gene as a new driver in the 11q13.3 amplicon in laryngeal/pharyngeal cancer. Clin Cancer Res, 13(21): 6257-66.

34. He, L., et al. (2016) Fas-associated protein with death domain (FADD) regulates autophagy through promoting the expression of Ras homolog enriched in brain (Rheb) in human breast adenocarcinoma cells. Oncotarget, 7(17): 24572-84. 
35. Pattje, W.J., et al. (2013) FADD expression is associated with regional and distant metastasis in squamous cell carcinoma of the head and neck. Histopathology, 63(2): 263-70.

36. Schrijvers, M.L., et al. (2012) FADD expression as a prognosticator in early-stage glottic squamous cell carcinoma of the larynx treated primarily with radiotherapy. Int J Radiat Oncol Biol Phys, 83(4): 1220-6.

37. Zhang, R., et al. (2017) The role of FADD in pancreatic cancer cell proliferation and drug resistance. Oncol Lett, 13(3): 1899-904.

38. Cimino, Y., et al. (2012) FADD protein release mirrors the development and aggressiveness of human non-small cell lung cancer. Br J Cancer, 106(12): 1989-96.

39. Schattenberg, J.M., et al. (2011) Cell death and hepatocarcinogenesis: Dysregulation of apoptosis signaling pathways. J Gastroenterol Hepatol, 26 Suppl 1: 213-9.

40. Tourneur, L., et al. (2003) Loss of FADD protein expression results in a biased Fas-signaling pathway and correlates with the development of tumoral status in thyroid follicular cells. Oncogene, 22(18): 2795-804.

41. Bhojani, M.S., et al. (2005) Nuclear localized phosphorylated FADD induces cell proliferation and is associated with aggressive lung cancer. Cell Cycle, 4(11): 1478-81.

42. Ikeda, T., et al. (2013) Phosphorylation status of Fas-associated death domain protein is associated with biochemical recurrence after radical prostatectomy. Urology, 81(3): 607-10.

43. Matsuyoshi, S., et al. (2006) FADD phosphorylation is critical for cell cycle regulation in breast cancer cells. Br J Cancer, 94(4): 532-9.

44. Drakos, E., et al. (2011) Expression of serine 194-phosphorylated Fas-associated death domain protein correlates with proliferation in B-cell non-Hodgkin lymphomas. Hum Pathol, 42(8): 111724. 
45. Patel, S., et al. (2014) Increased Expression of Phosphorylated FADD in Anaplastic Large Cell and Other T-Cell Lymphomas. Biomark Insights, 9: 77-84.

46. Khan, A.P., et al. (2010) High-throughput molecular imaging for the identification of FADD kinase inhibitors. J Biomol Screen, 15(9): 1063-70.

47. Tourneur, L., et al. (2005) FADD adaptor in cancer. Med Immunol, 4(1): 1. 


\section{FIGURE LEGENDS}

Fig. 1. FADD and S194-P-FADD reduction in T-LBL. (A) Total FADD protein was determined in CTRL and T-LBL groups by IHC. The box-and-whisker plot analysis (left) shows the positivity - in percentage of total FADD protein. Kernel density plot (right) shows T-LBLs density for FADD (dashed line) and standard normal distribution of each cluster (continuous line). (B, C) FADD and S194-P-FADD levels in CTRL, High and Low T-LBL samples, determined by IHC. Representative images are shown for each group (left). The box-and-whisker plot analyses (right) show the positivity - in percentage - of total FADD or S194-P-FADD protein.

The statistical significance of the comparisons is indicated as follows: ns, not significant; ${ }^{\star \star \star}, P \leq 0.001$.

Fig. 2. Subcellular localization of S194-P-FADD and FADD phospho-mutants. (A) The scatter plot analyses (left) show cytoplasmic (black triangles) and nuclear (white circles) S194-P-FADD positivity in CTRL, High and Low T-LBL samples, as determined by IHC. The statistical significance of every comparison is indicated. To illustrate the subcellular localization of S194-P-FADD, a representative image acquired at 100x magnification is shown (right). The black arrowhead illustrates a cell with nuclear positivity, the black and white arrowhead illustrates a cell with cytoplasmic positivity, and the open arrowhead illustrates negative cells. (B) Subcellular distribution of FADD was determined by WB in cytoplasmic and nuclear fractions from cell lines either deficient for FADD (NEG) or expressing wildtype FADD (FADD) or phospho-mutants (S194A and S194D). HSP90 indicated equivalent loading of samples and PARP served as a specific control for nuclear fraction. A representative image is shown (left). Reference protein weights in kilo Daltons are shown on the left of each blot. WB images are cropped in favour of conciseness. Densitometry (right; $n=6$ ) shows FADD protein abundance, normalized to HSP90 and referred to FADD cell line in cytoplasm and nucleus. Error bars represent SEM.

The statistical significance of the comparisons is indicated as follows: ns, not significant; ${ }^{*}, P \leq 0.05 ;{ }^{* *}, P$ $\leq 0.01 ;{ }^{* * *}, P \leq 0.001$. 
Fig. 3. FADD protein stability. (A) Cell lines expressing wildtype FADD or phospho-mutants were treated with $40 \mu \mathrm{g} / \mathrm{ml} \mathrm{CHX}$ for $0,2,4$ and 6 hours to determine FADD protein stability by WB. A representative image is shown (left). The relative amounts of FADD protein, normalized to $\alpha$-Tubulin, were calculated from three independent experiments and represented along time (right) for each cell line: blue, red and black represent FADD, S194A and S194D cell lines, respectively. (B) The same cell lines were treated with $40 \mu \mathrm{g} / \mathrm{ml} \mathrm{CHX}$ plus $10 \mu \mathrm{M} \mathrm{MG}-132$ for $6 \mathrm{~h}$ to determine proteasome involvement by WB.

Reference protein weights in kilo Daltons are shown on the left of each blot. WB images are cropped in favour of conciseness.

The statistical significance of the comparisons is indicated as follows: ${ }^{*}, P \leq 0.05$.

Fig. 4. Evaluation of proliferation in T-LBL samples. (A) Ki67 levels are determined by IHC in CTRL, High and Low T-LBL samples. Representative images are shown for each group (left). The box-and-whisker plot analyses (right) show the positivity - in percentage - of Ki67. (B) GSEA analysis in High vs. Low TLBL sub-groups shows a significant enrichment in the High T-LBL sub-group for the signature of T cell proliferation defined by the accession reference "Aviv Regev" (Broad Institute), Module 521. (C) Correlation of Ki67 with nuclear-S194-P-FADD, indicating the coefficient of determination $\left(\mathrm{R}^{2}\right)$ and the $\mathrm{P}$ value of regression analysis.

The statistical significance of the comparisons is indicated as follows: ${ }^{* *}, P \leq 0.01 ;{ }^{* * *}, P \leq 0.001$.

Fig. 5. Functional assays to evaluate FADD phosphorylation dependency of cell proliferation. (A) XTT cell proliferation assay $(n=4)$ was performed in NEG, FADD, S194A and S194D cell lines. The absorbance on the left axis results from subtracting background absorbance at $655 \mathrm{~nm}$ to formazanspecific absorbance at $490 \mathrm{~nm}$ and directly correlates to the number of viable cells (right axis). (B) S10-P- 
Histone-H3 determination by FC $(n=4)$ in the same cell lines, as a marker for mitosis. (C) Graph plotting the $2 n / 4 n$ ratio, normalized to that of $t=0$, as determined by DNA content FC of cells harvested every 20 min following release from nocodazole $\mathrm{G} 2 / \mathrm{M}$ block $(\mathrm{n}=6)$.

Error bars represent SEM. The statistical significance of the comparisons is indicated as follows: ${ }^{*}, P \leq$ $0.05 ; * * *, P \leq 0.001$

Fig. 6. FADD phosphorylation associates with tumour aggressiveness. (A) The box-and-whisker plot analysis of S194-P-FADD regarding the tumour stage, as defined previously [7], the involvement of extralymphatic organs and the recurrence status of the patient. NR, non-relapse; $\mathrm{Pr} / \mathrm{Re}$, progressive / recurrent. (B) GSEA analysis in High vs. Low T-LBL sub-groups shows a significant enrichment in the High samples for an oncogenic signature of 30 genes associated to T-cell lymphoblastic neoplasms. This signature was created ad hoc on the basis of previous literature [3] and is defined in Supplementary Table 3. The statistical significance of the comparisons is as follows: ns, not significant; ${ }^{*}, P \leq 0.05$. (C) Proposed model of association between FADD phosphorylation and prognosis. Tumour $\mathrm{T}$ cells from $\mathrm{T}$ LBL patients may exhibit high or low S194-P-FADD levels (diagnosis). The phosphorylated protein favours tumour T cell proliferation, thus rendering the tumour clinically more aggressive and impacting on the prognosis. 
Figure 1.

A

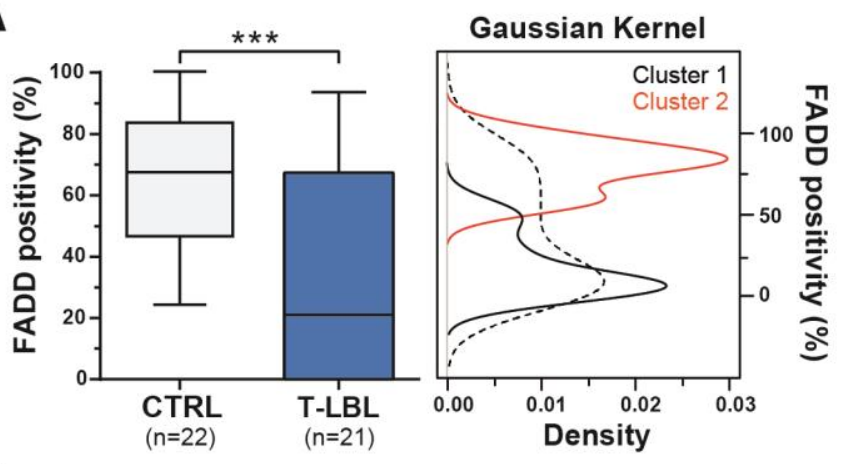

B
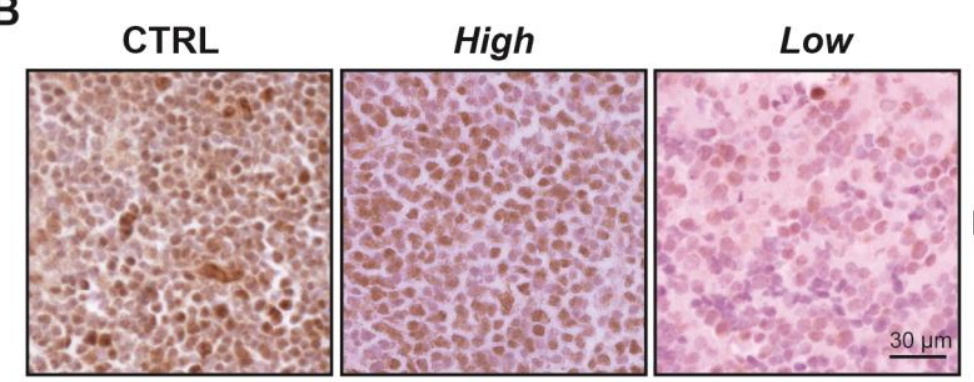

FADD

C
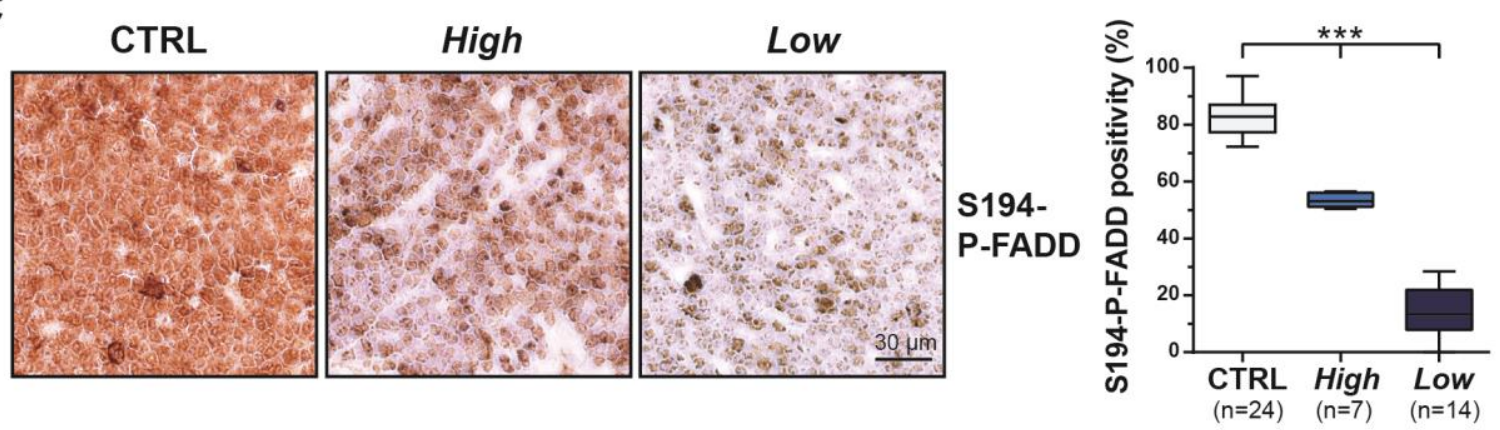

Figure 2.

A

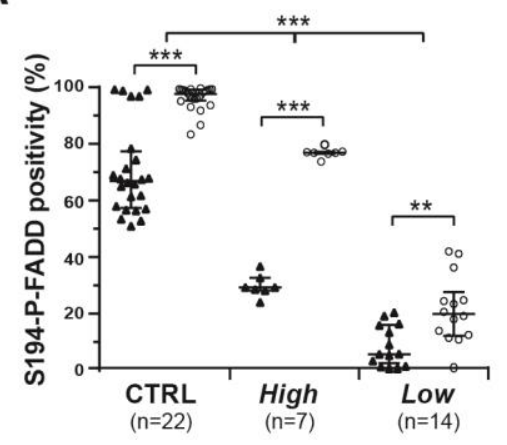

B

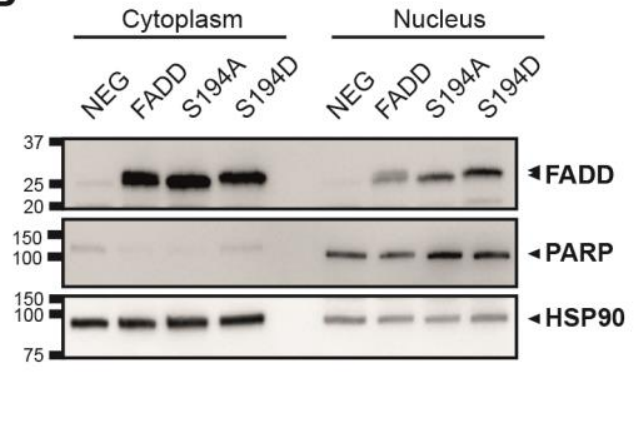

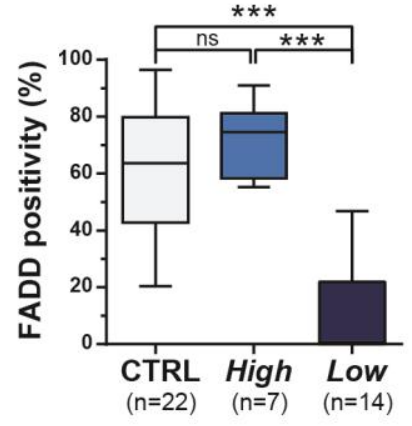

^ Cytoplasm
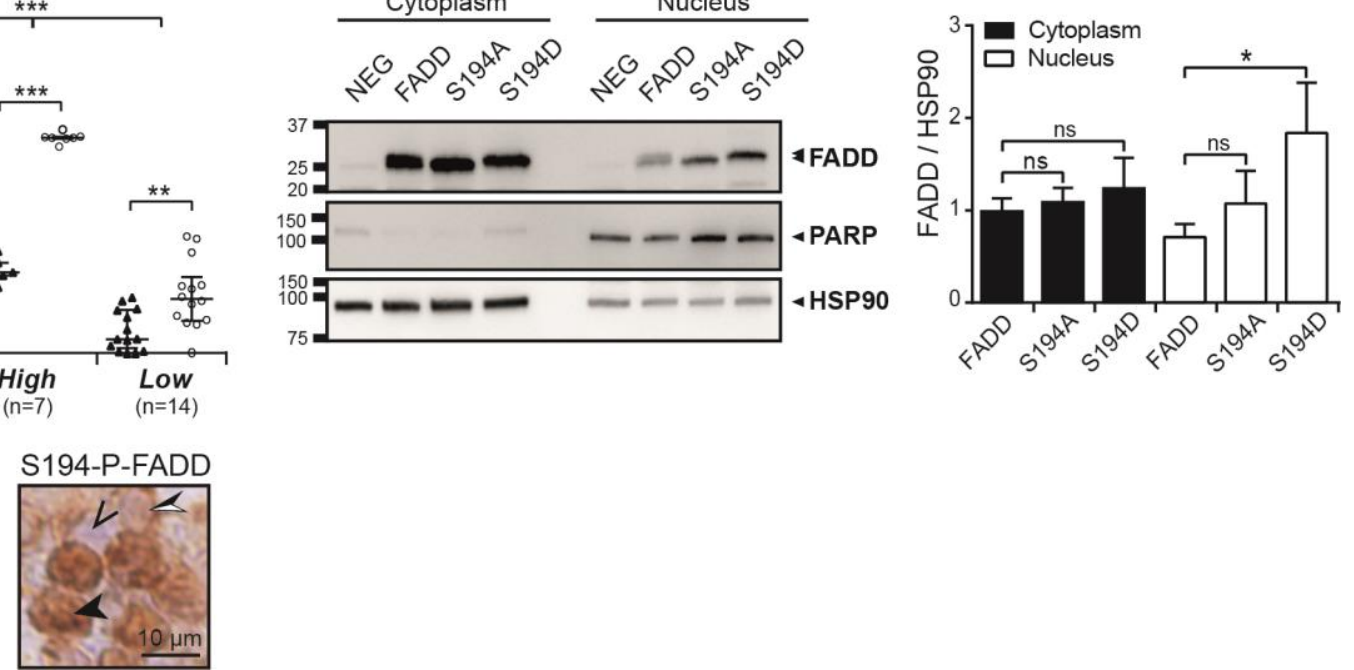

- Nucleus 
Figure 3.

A
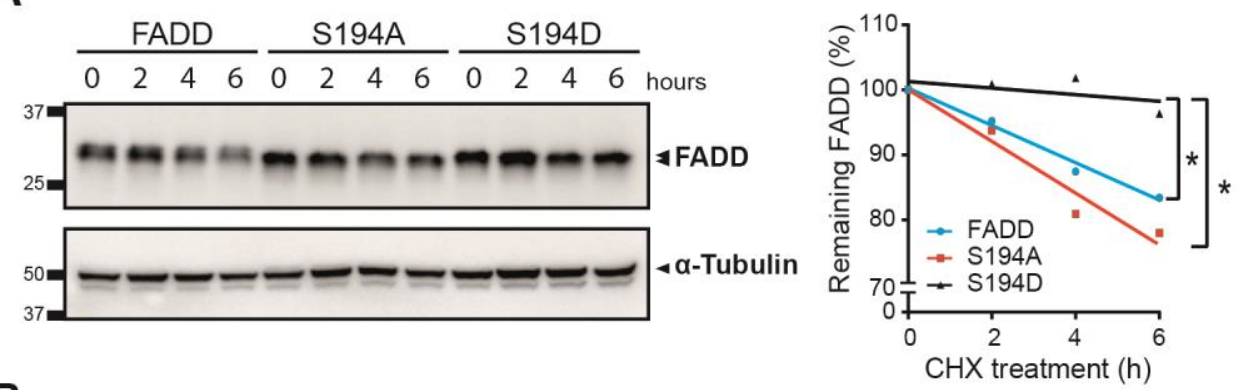

B

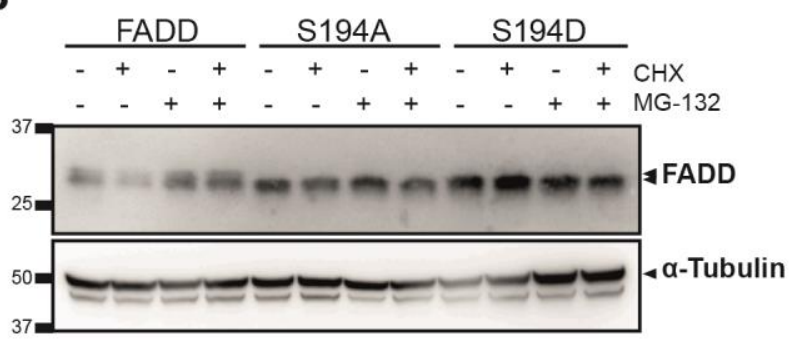

\section{Figure 4.}

A

CTRL

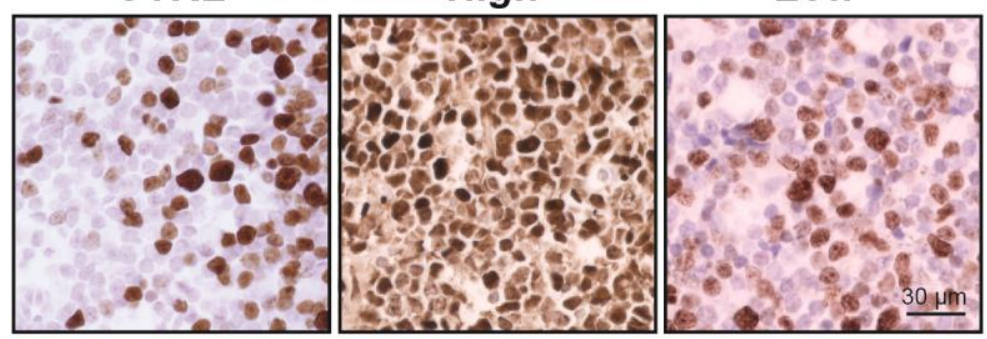

B

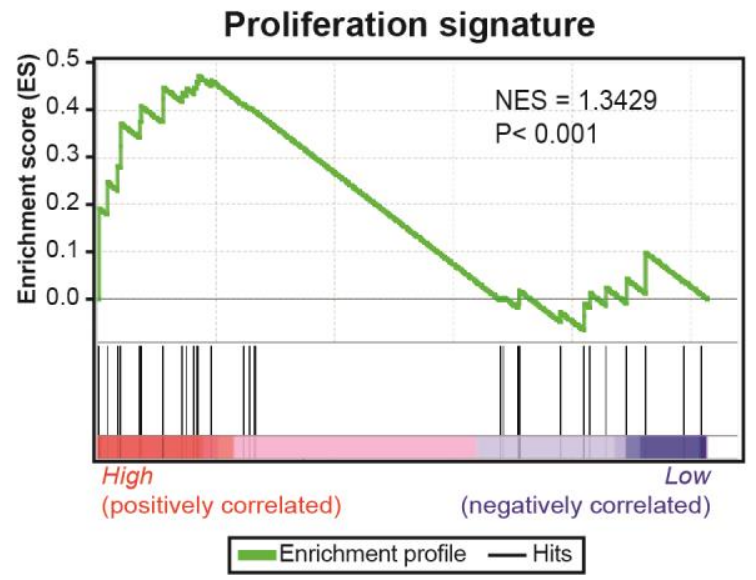

C
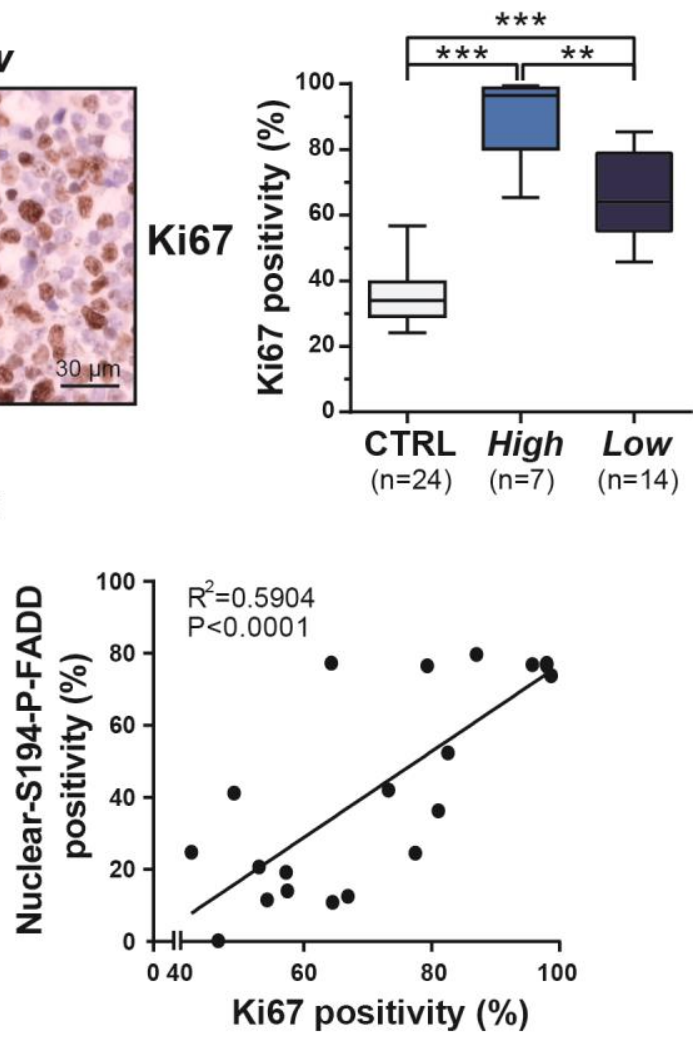
Figure 5.

A

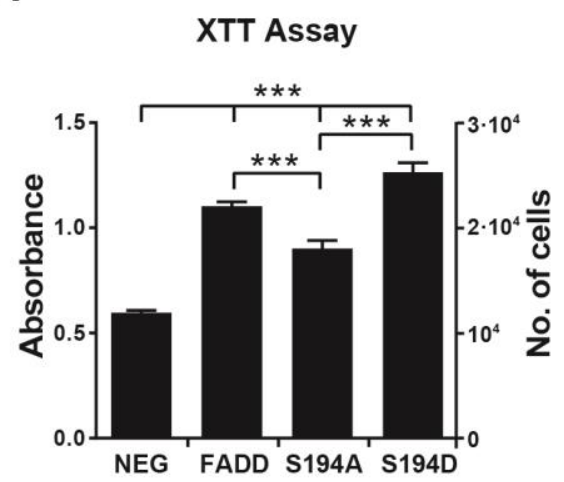

B

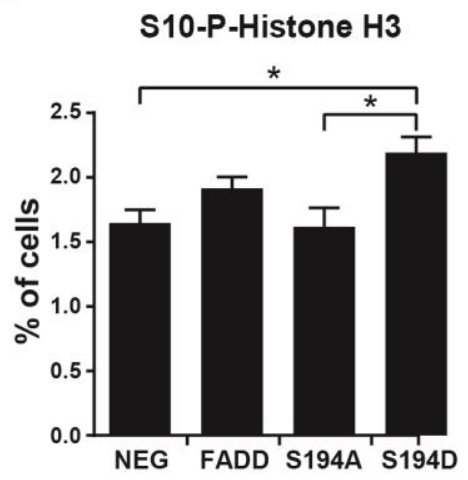

C

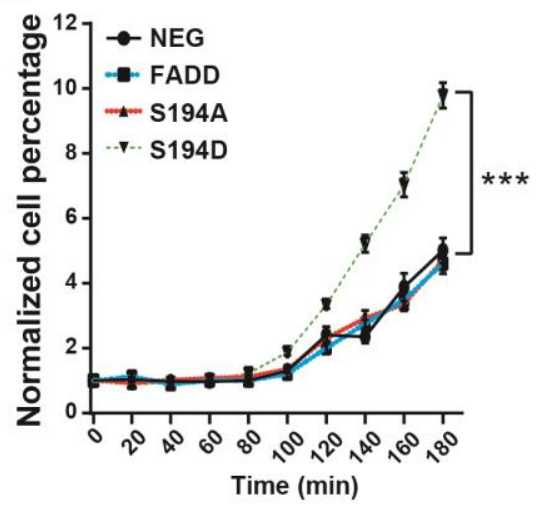

Figure 6.

A

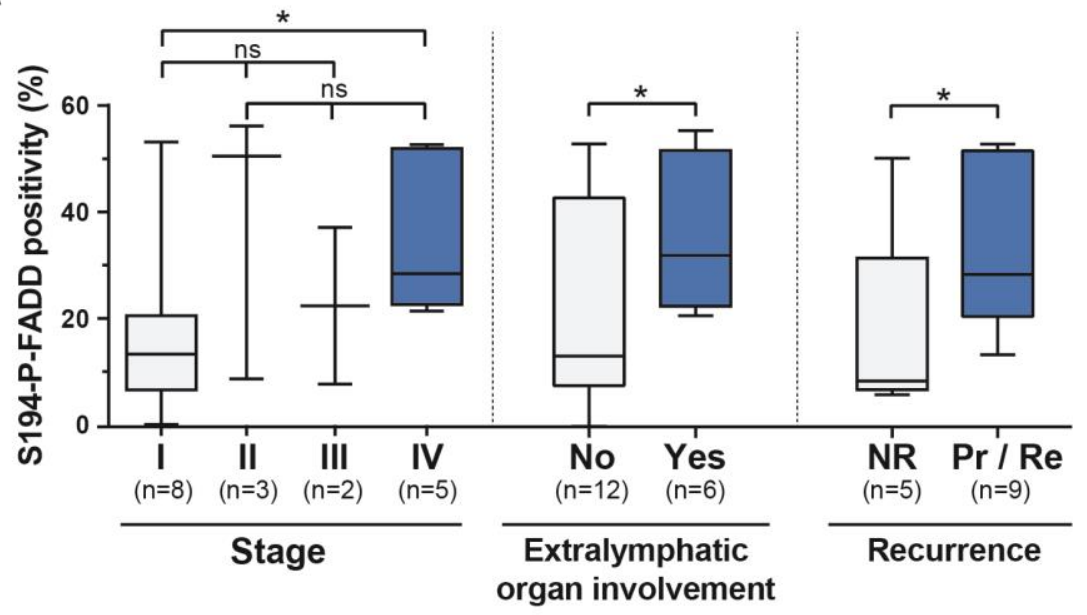

B

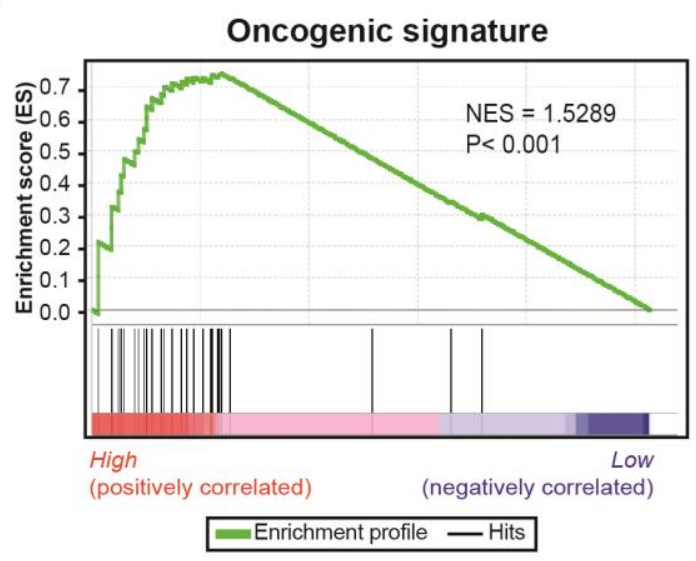

C

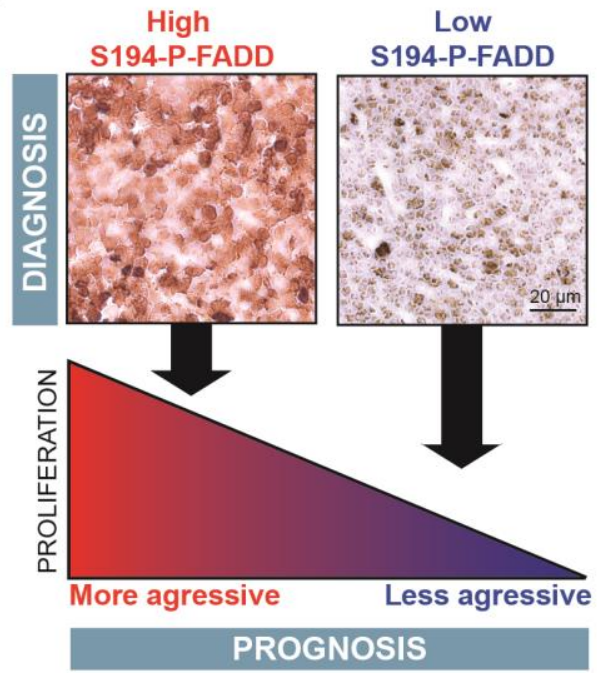

